\title{
ON DERIVATIONS IN PRIME RINGS AND BANACH ALGEBRAS
}

\author{
J. VUKMAN
}

(Communicated by Maurice Auslander)

\begin{abstract}
Let $R$ be a ring with center $Z(R)$. A mapping $F: R \rightarrow R$ is said to be centralizing on $R$ if $[F(x), x] \in Z(R)$ holds for all $x \in R$. The main purpose of this paper is to prove the following result, which generalizes a classical result of Posner: Let $R$ be a prime ring of characteristic not 2, 3, and 5. Suppose there exists a nonzero derivation $D: R \rightarrow R$, such that the mapping $x \mapsto[[D(x), x], x]$ is centralizing on $R$. In this case $R$ is commutative. Combining this result with some well-known deep results of Sinclair and Johnson, we generalize Yood's noncommutative extension of the Singer-Wermer theorem.
\end{abstract}

\section{PRELIMINARIES}

This paper is a continuation of our earlier work [17]. Throughout, $R$ represents an associative ring with center $Z(R)$. We write $[x, y]$ for $x y-y x$, and use the identities $[x y, z]=[x, z] y+x[y, z],[x, y z]=[x, y] z+y[x, z]$. Recall that $R$ is prime if $a R b=(0)$ implies that either $a=0$ or $b=0$, and is semiprime if $a R a=(0)$ implies $a=0$. An additive mapping $D$ from $R$ to $R$ is called a derivation if $D(x y)=D(x) y+x D(y)$ holds for all $x, y \in R$. A derivation $D$ is inner if there exists $a \in R$, such that $D(x)=[a, x]$ holds for all $x \in R$. An additive mapping $D$ from $R$ to $R$ is called a Jordan derivation if $D\left(x^{2}\right)=D(x) x+x D(x)$ holds for all $x \in R$. Obviously, every derivation is a Jordan derivation. The converse is in general not true. Herstein [7] has proved that every Jordan derivation on a prime ring of characteristic not two is a derivation. A brief proof of Herstein's result can be found in [4]. Cursack [6] has generalized Herstein's result on 2-torsionfree (i.e., such that $2 x=0$ implies $x=0$ ) semiprime rings (see also [2]). A mapping $F$ from $R$ to $R$ is said to be commuting on $R$ if $[F(x), x]=0$ holds for all $x \in R$, and is said to be centralizing on $R$ if $[F(x), x] \in Z(R)$ holds for all $x \in R$. For results concerning commuting, centralizing, and related mappings in prime and semiprime rings, we refer to $[1,5,9,10,16,17]$ where further references can be found.

Received by the editors March 20, 1990 and, in revised form, October 4, 1990.

1980 Mathematics Subject Classification (1985 Revision). Primary 16A12, 16A68, 16 A72.

Key words and phrases. Prime ring, derivation, Jordan derivation, inner derivation, commuting mapping, centralizing mapping, Banach algebra.

This research has been supported by the Research Council of Slovenia. 


\section{THE RESULTS}

A classical result in the theory of centralizing mappings is a theorem of Posner [11], which states that the existence of a nonzero centralizing derivation on a prime ring forces the ring to be commutative. In our recent paper [17] we have proved that in case there exists a nonzero derivation $D: R \rightarrow R$, where $R$ is a prime ring of characteristic different from 2 and 3, such that the mapping $x \mapsto[D(x), x]$ is centralizing on $R, R$ is commutative. Neglecting the fact that in our result we have an additional assumption concerning the characteristic of the ring, we can say that Theorem 2 in [17] generalizes Posner's theorem mentioned above. It is our aim in this paper to generalize Theorem 2 in [17] by proving the following result.

Theorem 1. Let $R$ be a noncommutative prime ring of characteristic different from 2, 3, and 5. Suppose there exists a derivation $D: R \rightarrow R$ such that the mapping $x \mapsto[[D(x), x], x]$ is centralizing on $R$. In this case $D=0$.

Proof. We introduce a mapping $B(\cdot, \cdot): R \times R \rightarrow R$ by the relation

$$
B[x, y]=[D(x), y]+[D(y), x], \quad x, y \in R .
$$

Obviously, $B(\cdot, \cdot)$ is symmetric (i.e., $B(x, y)=B(y, x)$ for all $x, y \in R$ ) and additive in both arguments. A routine calculation shows that the relation

$$
B(x y, z)=B(x, z) y+x B(y, z)+D(x)[y, z]+[x, z] D(y)
$$

holds for all $x, y, z \in R$. We also introduce a mapping $f$ from $R$ to $R$ by $f(x)=B(x, x)$. We have

$$
f(x)=2[D(x), x], \quad x \in R .
$$

Obviously, the mapping $f$ satisfies the relation

$$
f(x+y)=f(x)+f(y)+2 B(x, y), \quad x, y \in R .
$$

Throughout the proof, we use the mapping $B(\cdot, \cdot)$ and the relations (1), (2), and (3) without specific reference. The assumption of the theorem can now be written in the form

$$
\text { [[ } f(x), x], x] \in Z(R), \quad x \in R .
$$

First we intend to prove that the mapping $x \mapsto[f(x), x]$ is commuting on $R$. In other words, we are going to prove that

$$
[[f(x), x], x]=0
$$

holds for all $x \in R$. The linearization of (4) gives

$$
\begin{aligned}
& {[[f(y), x], x]+2[[B(x, y), x], x]+[[f(x), y], x]+[[f(y), y], x]} \\
& \quad+2[[B(x, y), y], x]+[[f(x), x], y]+[[f(y), x], y] \\
& \quad+2[[B(x, y), x], y]+[[f(x), y], y]+2[[B(x, y), y], y] \in Z(R) .
\end{aligned}
$$

Substituting $-x$ for $x$ in the above relation and comparing the new relation with the above relation, we obtain

$$
\begin{aligned}
& 2[[B(x, y), x], x]+[[f(x), y], x]+[[f(y), y], x]+[[f(x), x], y] \\
& \quad+[[f(y), x], y]+2[[B(x, y), y], y] \in Z(R) .
\end{aligned}
$$


Substituting $2 x$ instead of $x$ in (6), comparing the relation so obtained with (6), and using the fact that we have assumed that $R$ is of characteristic not 3, we obtain

(7) $[[f(x), x], y]+[[f(x), y], x]+2[[B(x, y), x], x] \in Z(R), \quad x, y \in R$.

Substituting $x^{2}$ instead of $y$ in (7), one obtains easily [[ $\left.\left.f(x), x\right], x\right] x \in Z(R)$, $x \in R$, which together with (4) gives

$$
[[f(x), x], x][x, y]=0, \quad x, y \in R .
$$

From (8) and Lemma 1 in [11], one can conclude that for any fixed $x \notin Z(R)$, we have $[[f(x), x], x]=0$ (note that for any fixed $x \notin \cdot Z(R)$ a mapping $y \mapsto[x, y]$ is a nonzero inner derivation), which proves the relation (5). From (5) we obtain

(9) $[[f(x), x], y]+[[f(x), y], x]+2[[B(x, y), x], x]=0, \quad x, y \in R$, in the same fashion that makes it possible to obtain (7) from (4). Let $y$ be $x y$ in (9). Then using (5) and (9), after some calculations we obtain

$$
\begin{aligned}
& 3[f(x), x][y, x]+2 f(x)[[y, x], x] \\
& \quad+D(x)[[[y, x], x], x]=0, \quad x, y \in R .
\end{aligned}
$$

Similarly, one obtains the relation

$$
\begin{aligned}
& 3[y, x][f(x), x]+2[[y, x], x] f(x) \\
& \quad+[[[y, x], x], x] D(x)=0, \quad x, y \in R .
\end{aligned}
$$

Putting $y=2 D(x)$ in (10) and (11), we arrive at

$$
3[f(x), x] f(x)+2 f(x)[f(x), x]=0, \quad x \in R,
$$

and

$$
3 f(x)[f(x), x]+2[f(x), x] f(x)=0, \quad x \in R .
$$

From (12) and (13) it follows immediately that $5[f(x), x] f(x)+5 f(x)[f(x), x]$ $=0, x \in R$, which gives $[f(x), x] f(x)+f(x)[f(x), x]=0, x \in R$, because we have assumed that $R$ is of characteristic not 5. Now $[f(x), x] f(x)=$ $-f(x)[f(x), x], x \in R$, together with (12) gives

$$
f(x)[f(x), x]=0, \quad x \in R,
$$

and

$$
[f(x), x] f(x)=0, \quad x \in R .
$$

From (14) we obtain

$$
\begin{aligned}
f(x) & {[f(x), y]+2 f(x)[B(x, y), x] } \\
+ & 2 B(x, y)[f(x), x]=0, \quad x, y \in R,
\end{aligned}
$$

using the same approach as in the proof of (7). Put $y x$ instead of $y$ in (16). Then

$$
\begin{aligned}
0= & f(x)[f(x), y x]+2 f(x)[B(x, y) x+y f(x)+[y, x] D(x), x] \\
& +2(B(x, y) x+y f(x)+[y, x] D(x))[f(x), x] \\
= & f(x)[f(x), y] x+f(x) y[f(x), x]+2 f(x)[B(x, y), x] x \\
& +2 f(x)[y, x] f(x)+2 f(x) y[f(x), x]+2 f(x)[[y, x], x] D(x) \\
& +f(x)[y, x] f(x)+2 B(x, y) x[f(x), x]+2 y f(x)[f(x), x] \\
& +2[y, x] D(x)[f(x), x] .
\end{aligned}
$$


According to (16), one can write $-2 B(x, y)[f(x), x] x$ in the above calculation instead of $f(x)[f(x), y] x+2 f(x)[B(x, y), x] x$. Now by (5) and (14), we have

$$
\begin{aligned}
& 3 f(x) y[f(x), x]+3 f(x)[y, x] f(x)+2 f(x)[[y, x], x] D(x) \\
& \quad+2[y, x] D(x)[f(x), x]=0,
\end{aligned}
$$

which can be written in the form

$$
\begin{aligned}
& 3[f(x), y][f(x), x]+3 f(x)[y, x] f(x)+2 f(x)[[y, x], x] D(x) \\
& \quad+2[y, x] D(x)[f(x), x]=0, \quad x, y \in R .
\end{aligned}
$$

Substituting $y z$ for $y$ in (17), after some calculations and similar substitutions as in the proof of (17) we obtain

$$
\begin{aligned}
& 3[f(x), y] z[f(x), x]+3[f(x), y][z, x] f(x)+2[f(x), y][[z, x], x] D(x) \\
& \quad+3 f(x)[y, x] z f(x)+2 f(x)[[y, x], x] z D(x) \\
& \quad+4 f(x)[y, x][z, x] D(x)+2[y, x] z D(x)[f(x), x]=0
\end{aligned}
$$

and in particular for $y=f(x)$,

$$
[f(x), x] y D(x)[f(x), x]=0, \quad x, y \in R,
$$

which gives

$$
D(x)[f(x), x]=0, \quad x \in R,
$$

by primeness of $R$. In the same fashion one can prove the relation

$$
[f(x), x] D(x)=0, \quad x \in R,
$$

starting from (15). From (18) and (19) one obtains

$$
D(x)[f(y), y]+D(y)[f(y), x]+2 D(y)[B(x, y), y]=0, \quad x, y \in R,
$$

and

$$
[f(y), y] D(x)+[f(y), x] D(y)+2[B(x, y), y] D(y)=0, \quad x, y \in R .
$$

Substituting $x y$ for $x$ in (20), one obtains

$$
\begin{aligned}
& 3[D(y), x][f(y), y]+3 D(y)[x, y] f(y) \\
& \quad+2 D(y)[[x, y], y] D(y)=0, \quad x, y \in R .
\end{aligned}
$$

Similarly, (21) gives

$$
\begin{aligned}
& 3[f(y), y][x, D(y)]+3 f(y)[x, y] D(y) \\
& \quad+2 D(y)[[x, y], y] D(y)=0, \quad x, y \in R .
\end{aligned}
$$

Now the substitution $x z$ for $x$ in (22) leads to

$$
\begin{aligned}
& 3[D(y), x] z[f(y), y]+3[D(y), x][z, y] f(y)+2[D(y), x][[z, y], y] D(y) \\
& \quad+3 D(y)[x, y] z f(y)+2 D(y)[[x, y], y] z D(y) \\
& \quad+4 D(y)[x, y][z, y] D(y)=0, \quad x, y \in R .
\end{aligned}
$$


Similarly one can prove the relation

(25)

$$
\begin{aligned}
& 3[f(y), y] z[x, D(y)]+3 f(y)[z, y][x, D(y)]+2 D(y)[[z, y], y][x, D(y)] \\
& \quad+3 f(y) z[x, y] D(y)+2 D(y) z[[x, y], y] D(y) \\
& \quad+4 D(y)[z, y][x, y] D(y)=0, \quad x, y \in R
\end{aligned}
$$

putting $z x$ instead of $x$ in (23). In particular, for $x=2 D(y),(24)$ and (25) reduce to

$$
3 D(x) f(x) y f(x)+4 D(y) f(x)[y, x] D(x)=0, \quad x, y \in R,
$$

and

$$
3 f(x) y f(x) D(x)+4 D(x)[y, x] f(x) D(x)=0, \quad x, y \in R .
$$

Replacing $y$ by $y D(x)$ in (26), we arrive at

$$
3 D(x) f(x) y D(x) f(x)+4 D(x) f(x)[y, x] D(x)^{2}+2 D(x) f(x) y f(x) D(x)=0
$$

for all $x, y \in R$. On the other hand, right multiplication of (26) by $D(x)$ leads to

$$
3 D(x) f(x) y f(x) D(x)+4 D(x) f(x)[y, x] D(x)^{2}=0, \quad x, y \in R .
$$

Combining (28) with (29), we arrive at

$$
3 D(x) f(x) y D(x) f(x)-D(x) f(x) y f(x) D(x)=0, \quad x, y \in R .
$$

Similarly, one obtains the relation

$$
3 f(x) D(x) y f(x) D(x)-D(x) f(x) y f(x) D(x)=0, \quad x, y \in R,
$$

starting from (27). Combining (30) with (31), one obtains

$$
D(x) f(x) y D(x) f(x)=f(x) D(x) y f(x) D(x), \quad x, y \in R .
$$

Now it follows from (32) and Theorem 7 in [2] that for any fixed $x \in R$ we have either $D(x) f(x)=f(x) D(x)$ or $D(x) f(x)=-f(x) D(x)$. In both cases, the relation (31) reduces to $f(x) D(x) y f(x) D(x)=0, x, y \in R$, which gives

$$
f(x) D(x)=0, \quad x \in R,
$$

by primeness of $R$. Of course we have also

$$
D(x) f(x)=0, \quad x \in R .
$$

From (33) one obtains

$$
f(x) D(y)+2 B(x, y) D(x)=0, \quad x, y \in R .
$$

Substitute $y z$ for $y$ in (35). Then after some calculations and suitable substitutions, we obtain $2 B(x, y)[z, D(x)]+[f(x), y] D(z)+2 D(y)[z, x] D(x)+$ $2[y, x] D(z) D(x)=0, x, y, z \in R$, and in particular, for $z=D(x)$,

$$
[f(x), y] D^{2}(x)+2[y, x] D^{2}(x) D(x)=0, \quad x, y \in R,
$$

since (33) holds. Substituting $y z$ for $y$ in (36) gives

$$
[f(x), y] z D^{2}(x)+2[y, x] z D^{2}(x) D(x)=0, \quad x, y, z \in R .
$$


In particular, for $y=D(x)$ the relation (37) reduces to

$$
f(x) y D^{2}(x) D(x)=0, \quad x, y \in R,
$$

because (33) and (34) hold. Let us assume that $D^{2}(a) D(a) \neq 0$ for some $a \in R$. In this case it follows from (38) that $f(a)=0$. Hence (37) reduces to $[y, a] z D^{2}(a) D(a)=0$, which implies $a \in Z(R)$, since we have assumed that $D^{2}(a) D(a) \neq 0$. In other words, we have proved that $D^{2}(x) D(x)=0$ for any $x \neq Z(R)$. We intend to prove that

$$
D^{2}(x) D(x)=0
$$

for all $x \in R$. Therefore, let $x$ be from $Z(R)$ and let $y \neq Z(R)$. We also have $x+y \neq Z(R)$. We know that $D^{2}(y) D(y)=0$ and $D^{2}(x+y) D(x+y)=0$, whence it follows $D^{2}(x) D(x)+D^{2}(x) D(y)+D^{2}(y) D(x)=0$. Substituting $-x$ for $x$ and comparing both relations, we obtain (39). The linearization of (39) gives $D^{2}(x) D(y)+D^{2}(y) D(x)=0, x, y \in R$. Substituting $y z$ for $y$, one obtains easily $D^{2}(y)[z, D(x)]+\left[D^{2}(x), y\right] D(z)+2 D(y) D(z) D(x)=0, x, y$, $z \in R$, and in particular, for $z=D(x)$,

$$
\left[D^{2}(x), y\right] D^{2}(x)=0, \quad x, y \in R .
$$

For any fixed $x \in R$ the mapping $y \mapsto\left[D^{2}(x), y\right]$ is an inner derivation. Hence from (40) and Lemma 1 in [11] it follows that for any fixed $x \in R$ we have either $D^{2}(x)=0$ or $D^{2}(x) \in Z(R)$. In any case $D^{2}(x) \in Z(R)$ for all $x \in R$, which makes it possible to conclude that left multiplication of (39) by $y$ gives $D^{2}(x) y D(x)=0, x, y \in R$. From this relation it follows that for any $x \in R$, we have either $D^{2}(x)=0$ or $D(x)=0$ by primeness of $R$. In any case, $D^{2}(x)=0$ for all $x \in R$, which yields $D=0$ by Theorem 2 in [11]. The proof of the theorem is complete.

We feel that Theorem 1 can be proved without the assumption that $R$ is of characteristic different from 5 , but unfortunately we are unable to do it.

Theorem 1 leads to the following conjecture: Let $D: R \rightarrow R$ be a derivation, where $R$ is a noncommutative prime ring with suitable characteristic restrictions. Suppose that for some integer $n$ we have $f_{n}(x)=0$ for all $x \in R$, where $f_{1}(x)=[D(x), x]$ and $f_{n+1}(x)=\left[f_{n}(x), x\right]$. In this case $D=0$. We feel that this conjecture would be hard to prove for arbitrary $n$, and would almost certainly require higher-powered methods than those used in the proof of Theorem 1.

Singer and Wermer [14] have proved that any continuous linear derivation on a commutative Banach algebra maps the algebra into its radical. Thomas [15] has proved this result without assuming the continuity of derivation. Yood [18] has extended the Singer-Wermer theorem on noncommutative Banach algebras by proving that every continuous linear derivation $D$ of a Banach algebra $A$, which satisfies $[D(x), y] \in \operatorname{rad}(A)$ for all $x, y \in A$, where $\operatorname{rad}(A)$ denotes the radical of $A$, maps $A$ into $\operatorname{rad}(A)$. We now generalize Yood's result as follows.

Theorem 2. Let $A$ be a noncommutative Banach algebra, and let $D: A \rightarrow A$ be a continuous linear Jordan derivation. If $[[[D(x), x], x], x] \in \operatorname{rad}(A)$ for all $x \in A$, then $D$ maps $A$ into $\operatorname{rad}(A)$. 
Proof. By Lemma 3.2 in Sinclair's paper [13], every continuous linear Jordan derivation $D$ of a Banach algebra $A$ leaves the primitive ideals of $A$ invariant. Since the radical of $A$ is the intersection of all primitive ideals, we have $D(\operatorname{rad}(A)) \subset \operatorname{rad}(A)$, which means that there is no loss of generality in assuming that $A$ is semisimple. Since $D$ leaves all primitive ideals invariant, one can introduce for any primitive ideal $P \subset A$ a Jordan derivation $D_{P}: A / P \rightarrow A / P$, where $A / P$ is the factor algebra, by $D_{P}(\hat{x})=D(x), \hat{x}=x+P$. The factor algebra $A / P$ is prime, since $P$ is a primitive ideal. Hence by Herstein's result $D_{P}$ is a derivation. The assumption of the theorem $[[[D(x), x], x], x]=0$, $x \in R$, gives $\left[\left[\left[D_{P}(\hat{x}), \hat{x}\right], \hat{x}\right], \hat{x}\right]=0, \hat{x} \in A / P$. Hence, in case $A / P$ is noncommutative, we have $D_{P}=0$, since all the assumptions of Theorem 1 are fulfilled. It remains to prove that $D_{P}=0$ also in the case when $A / P$ is commutative. Johnson and Sinclair [8] have proved that any linear derivation on a semisimple Banach algebra is continuous. Combining this result with the SingerWermer theorem, one obtains that there are no nonzero linear derivations on commutative semisimple Banach algebras. Hence in case $A / P$ is commutative, we have $D_{P}=0$ as well. In other words $D(x)$ is in the intersection of all primitive ideals of $A$ for any $x \in A$. Since the intersection of all primitive ideals is the radical, and since $A$ is semisimple, we have $D=0$. The proof of the theorem is complete.

As we have mentioned above, Thomas [15] has generalized the Singer-Wermer theorem by proving that any linear derivation on a commutative Banach algebra maps the algebra into its radical. This result leads to the question of whether Theorem 2 can be proved without any continuity assumption. We do not know if the answer to this question is affirmative. However, in a special case, when a Banach algebra is semisimple, one can prove the following result.

Theorem 3. Let $A$ be a nomcommutative semisimple Banach algebra. Suppose there exists a linear Jordan derivation $D: A \rightarrow A$, such that the mapping $x \mapsto$ $[[D(x), x], x]$ is commuting on $A$. In this case $D=0$.

Proof. The proof goes through in the same way as the proof of Theorem 2 with the only exception that at the beginning of the proof one has to use the fact that any linear Jordan derivation on a semisimple Banach algebra is continuous (see [2, Theorem 6]).

\section{REFERENCES}

1. H. E. Bell and W. S. Martindale, Centralizing mappings of semiprime rings, Canad. Math. Bull. 30 (1987), 92-101.

2. M. Brešar, Jordan derivations on semiprime rings, Proc. Amer. Math. Soc. 104 (1988), 1003-1006.

3. M. Brešar and J. Vukman, Jordan derivations on prime rings, Bull. Austral. Math. Soc. 37 (1988), 321-322.

4. $\ldots$, On some additive mappings in rings with involution, Aequationes Math. 38 (1989), 178-185.

5. __ On left derivations and related mappings, Proc. Amer. Math. Soc. 110 (1990), 7-16.

6. J. Cusack, Jordan derivations on rings, Proc. Amer. Math. Soc. 53 (1975), 321-324.

7. I. N. Herstein, Jordan derivations on prime rings, Proc. Amer. Math. Soc. 8 (1957), 1104 1110. 
8. B. E. Johnson, Continuity of derivations on commutative Banach algebras, Amer. J. Math. 91 (1969), 1-10.

9. J. Mayne, Ideals and centralizing mappings in prime rings, Proc. Amer. Math. Soc. 86 (1982), 211-213; Erratum 89 (1983), 183.

10. __ Centralizing mappings of prime rings, Canad. Math. Bull. 27 (1984), 122-126.

11. E. Posner, Derivations in prime rings, Proc. Amer. Math. Soc. 8 (1957), 1093-1 100.

12. A. M. Sinclair, Continuous derivations on Banach algebras, Proc. Amer. Math. Soc. 20 (1969), 166-170.

13. Jordan homomorphisms and derivations on semisimple Banach algebras, Proc. Amer. Math. Soc. 24 (1970), 209-214.

14. I. M. Singer and J. Wermer, Derivations on commutative normed algebras, Math. Ann. 129 (1955), 260-264.

15. M. P. Thomas, The image of a derivation is contained in the radical, Ann. of Math. (2) 128 (1988), 435-460.

16. J. Vukman, Symmetric bi-derivations on prime and semiprime rings, Aequationes Math. 38 (1989), 245-254.

17. __ Commuting and centralizing mappings in prime rings, Proc. Amer. Math. Soc. 109 (1990), 47-52.

18. B. Yood, Continuous homomorphisms and derivations on Banach algebras, Contemp. Math., vol. 32, Amer. Math. Soc., Providence, RI, 1984, pp. 279-284.

Department of Mathematics, University of Maribor, PF, Koroska 160, 62000 Maribor, YUGOSLAVIA 